\title{
Desigualdades socioeconómicas en la mortalidad en el País Vasco y sus capitales: un análisis de áreas geográficas pequeñas (Proyecto MEDEA)
}

\section{Socioeconomic inequalities in mortality in the Basque Country and its main cities: a small geographical areas analysis (MEDEA Project)}

\author{
Santiago Esnaola ${ }^{1}$, Imanol Montoya ${ }^{2}$, Montse Calvo ${ }^{1}$, Berta Ibáñez ${ }^{3}$,

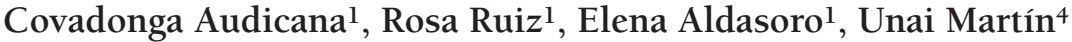

\section{INTRODUCCIÓN}

En los últimos años ha resurgido con fuerza el estudio del territorio como determinante de la salud, especialmente utilizando áreas geográficas pequeñas (Kawachi y Berkman, 2003). El análisis de la distribución geográfica de la salud en áreas pequeñas es útil para generar hipótesis etiológicas, estudiar el papel de los factores de riesgo, y servir de guía para la planificación sanitaria (English, 1992). Al estudiar las desigualdades socioeconómicas, el uso de áreas pequeñas permite tener en cuenta los atributos del área de residencia o contextuales como determinantes de la salud (Susser y Susser, 1996; DiezRoux, 2001). Además, la detección de áreas geográficas pequeñas con peores indicadores socioeconómicos y de salud posibilita una mejor orientación de las intervenciones.

\footnotetext{
${ }^{1}$ Departamento de Sanidad. Gobierno Vasco [sesnaola@ej-gv.es].

2 Unidad de investigación de atención primaria de Bizkaia.

${ }^{3}$ Fundación BIOEF.

${ }^{4}$ Universidad del País Vasco.
} 
Las evidencias disponibles sobre las desigualdades socioeconómicas en la mortalidad en áreas pequeñas son relativamente numerosas en el ámbito anglosajón (Philimore y Reading, 1992; Wallace y Wallace, 1997; Mcintyre y Elleway, 2000). En España, en los últimos años han proliferado los estudios en este ámbito de investigación. La mayoría de los relativos al conjunto de España (Benach et al., 1999; Benach et al., 2003) o a regiones (Benach et al., 2002) han utilizado municipios o agregados de municipios como unidad de análisis. Más numerosos han sido los dedicados a zonas urbanas, utilizando el barrio o la zona básica de salud como unidad de análisis, siendo la mayoría de ellos de la ciudad de Barcelona (Borrell y Arias, 1993; Pasarín et al., 1999), de Sevilla (Ruiz-Ramos et al., 2004), y Valencia y Alicante (Nolasco et al., 2004). Más recientemente se ha utilizado la sección censal como unidad de análisis en estudios relativos a las capitales de Andalucía (Ocaña et al., 2009) y Barcelona (Domínguez-Berjón et al., 2005; Cano-Serral et al., 2009).

Se han evidenciado las ventajas del uso de la sección censal como área geográfica para el estudio de la variabilidad espacial de resultados en salud y para el estudio de las desigualdades socioeconómicas en salud (Krieger, 2006). En ellas la población es más homogénea en cuanto a condiciones socioeconómicas, el entorno presenta características medioambientales similares, la información necesaria para llevar estudios ecológicos se encuentra habitualmente disponible en organismos oficiales y hay pocos problemas de confidencialidad asociados. Sin embargo, el uso de áreas pequeñas como unidades de análisis puede conllevar que muchas unidades geográficas tengan una población muy reducida. Por esa razón, la estimación de los indicadores de mortalidad puede ser muy inestable: el cambio en una o dos defunciones puede provocar grandes cambios en el valor estimado. Para hacer frente a este problema, pueden utilizarse métodos basados en un enfoque bayesiano, que combinan la información del área en cuestión con la del resto de las áreas o con un subconjunto de ellas (Clayton y Kaldor, 1987).

En el País Vasco un estudio previo relativo a todo el territorio describió las desigualdades socioeconómicas en la mortalidad usando la sección censal como unidad de análisis (Esnaola et al., 2006). No obstante, el análisis no incluyó a las capitales y los métodos de análisis no tuvieron en cuenta la estructura espacial de los datos. El objetivo del presente estudio fue describir las desigualdades socioeconómicas en la mortalidad por áreas pequeñas en el País Vasco y en sus tres capitales, utilizando la sección censal como unidad de análisis y métodos estadísticos adecuados para el análisis de áreas geográficas pequeñas. 


\section{MÉTODOS}

\section{Diseño, población y unidad de análisis}

Este estudio se realizó en el contexto del proyecto MEDEA (Desigualdades socioeconómicas y ambientales en la mortalidad en áreas pequeñas de ciudades españolas) relativo al País Vasco, que incluye todo el territorio de la CAPV. Se utilizó un diseño ecológico transversal. La población de estudio fue la residente en la CAPV en 2001 (2.082.587 habitantes según el Censo de 2001). Se incluyeron todas las defunciones ocurridas durante los años 1996-2003. Se tomó como unidad geográfica la seccion censal. Aquellas secciones con menos de 500 habitantes fueron incluidas en las secciones adyacentes. Finalmente, se estudiaron 1.645 secciones censales, con un tamaño poblacional medio de 1.257 habitantes, de las cuales 288 pertenecen a Bilbao, 140 a Donostia-San Sebastián y 168 a Vitoria-Gasteiz.

\section{Fuentes de información y variables de estudio}

Los datos sobre las defunciones, la población y los indicadores socioeconómicos se obtuvieron del Instituto Vasco de Estadística (Eustat). Los datos sobre el número de residentes en cada sección censal (por sexo y grupos quinquenales de edad) y sobre los indicadores socioeconómicos provienen del Censo de Población y Viviendas de 2001. Los relativos a las defunciones proceden de la Estadística de Defunciones de la CAPV realizada por Eustat en colaboración con el INE y el Departamento de Sanidad del Gobierno Vasco. Además de los datos de los Boletines Estadísticos de Defunción, esa Estadística recoge la información territorial de la residencia de la persona fallecida incluyendo la codificación del distrito y sección censales. Se obtuvo así el número de defunciones observadas por sexo, grupo quinquenal de edad y sección censal de residencia. No pudo geocodificarse el lugar de residencia en el 3,6\% de las defunciones. El número de defunciones esperadas en cada sexo, grupo quinquenal de edad y sección censal se calculó a partir de las tasas por sexo y grupo de edad de la CAPV para el año 2001.

\section{Indice de privación}

Se construyó un índice de privación para cada sección censal siguiendo la metodología del proyecto MEDEA (Domínguez-Berjón et al., 2008). Se utilizó 
el análisis de componentes principales para sintetizar la información recogida en cinco indicadores socioeconómicos relativos a las secciones censales: Proporción de desempleo en la población activa; proporción de instrucción insuficiente (población de 16 años o más analfabeta o con menos de 5 años de escolarización); proporción de instrucción insuficiente en jóvenes (población de 16 a 29 años analfabeta o con menos de 5 años de escolarización); proporción de trabajadores manuales en la población ocupada; y proporción de trabajadores con contrato eventual en la población ocupada. El índice fue normalizado con una media igual a cero y una desviación estándar de 1. El índice de privación fue calculado de forma separada para cada uno de los cuatro ámbitos geográficos (CAPV, Bilbao, Donostia-San Sebastián y Vitoria-Gasteiz). En el caso de la CAPV el índice de privación explicaba el 58\% de la variabilidad total de los indicadores socioeconómicos incluidos. En las capitales ese valor fue de 79\% para Bilbao, 67\% para Donostia-San Sebastián y 69\% para Vitoria-Gasteiz.

\section{Análisis estadístico}

Se utilizó la Razón de Mortalidad Estandarizada (RME) suavizada como estimador del riesgo relativo de mortalidad de cada sección censal. El número observado de defunciones $\left(\mathrm{O}_{\mathrm{i}}\right)$ para cada sección censal $(\mathrm{i}=1, \ldots, \mathrm{n}$, con $\mathrm{n}$ igual al número de secciones) sigue una distribución de Poisson con media $\mu_{\mathrm{i}}$ $=\mathrm{E}_{\mathrm{i}} \theta_{\mathrm{i}}$, donde $\mathrm{E}_{\mathrm{i}}$ representa el número esperado de defunciones para cada sección censal obtenido mediante estandarización indirecta, y $\theta_{\mathrm{i}}$ el riesgo relativo de cada área específica. El estimador de máxima verosimilitud de $\theta_{\mathrm{i}}$ fue la RME para la sección censal i: $\hat{\theta}_{i}=O_{i} / E_{i}$ (la razón entre el número observado y esperado de defunciones) con una varianza estimada de $O_{i} / E_{i}^{2}$, por lo que puede presentar una alta variabilidad en áreas con pequeñas poblaciones. Para solventar el problema de la inestabilidad de las RME se obtuvieron estimadores suavizados del riesgo utilizando el modelo jerárquico de regresión de Poisson de efectos aleatorios introducido por Clayton y Kaldor (Clayton y Kaldor, 1987), y desarrollado por Besag, York y Mollié (Besag, York y Mollié, 1991) (Modelo BYM), que incluye dos efectos aleatorios: 1) el espacial $\left(\mathrm{S}_{\mathrm{i}}\right)$, que introduce estructura espacial al modelo y permite a cada área aprovecharse de la información de las áreas vecinas, de manera que el valor esperado de dicho efecto aleatorio en cada área condicionado al resto de áreas es el promedio de este efecto en las zonas contiguas, y 2$)$ el heterogéneo o no estructurado $\left(\mathrm{H}_{\mathrm{i}}\right)$, que es independiente para cada área. Se asignan distribuciones previas a los 
efectos aleatorios, e hiper previas a los parámetros de las distribuciones previas. Para el efecto espacial se seleccionó un modelo normal condicional autoregresivo (CARN) cuya matriz de precisión es proporcional a la matriz de vecindades en tres secciones censales, considerando como vecinas aquellas áreas que compartieran sus fronteras, y una distribución normal de media cero y varianza $\alpha^{2}{ }_{h}$ para el efecto heterogéneo. Tal como sugieren Gelman et al. (Gelman et al., 2006), se asignó una distribución uniforme $\mathrm{U}(0,5)$ a la desviación estándar de los efectos aleatorios. El modelo asume que el logaritmo de la media de la mortalidad observada tiene la forma siguiente:

$\log \left(\mu_{\mathrm{i}}\right)=\log \left(\mathrm{E}_{\mathrm{i}}\right)+\alpha+\mathrm{S}_{\mathrm{i}}+\mathrm{H}_{\mathrm{i} ;}$ donde $\alpha$ es la constante.

Como extension del modelo anterior, se añadió el índice de privación $\left(\mathrm{X}_{\mathrm{ij}}\right)$ :

$\log \left(\mu_{\mathrm{i}}\right)=\log \left(\mathrm{E}_{\mathrm{i}}\right)+\alpha+\sum \beta_{\mathrm{j}} \mathrm{X}_{\mathrm{ij}}+\mathrm{S}_{\mathrm{i}}+\mathrm{H}_{\mathrm{i}}$; donde e es es el riesgo relativo (RR) de mortalidad de las secciones en el quintil j del índice de privación con respecto al primer quintil, más favorecido.

Las estimaciones del RR fueron valoradas mediante la mediana de la distribución posterior y sus intervalos de credibilidad del 95\%. La distribución fue obtenida mediante métodos Monte Carlo basados en cadenas de Markov (MCMC), usando el programa WINBUGS, versión 1.4.3 (Lunn et al., 2000), que fue utilizado a través del programa R 2.3.1 ( $\mathrm{R}$ Development Core Team, 2007). La convergencia del modelo fue valorada mediante el estadístico $\hat{R}$ (Estadística de Brooks-Gelman-Rubin en WINBUGS) y el tamaño muestral (estadístico n.eff en R) (Brooks y Gelman, 1998). Los criterios de convergencia fueron los consensuados en el proyecto MEDEA y previamente utilizados en el Atlas de Mortalidad de la Comunidad Valenciana (Martínez Beneito, 2005): $\hat{R}$ menor a 1,1 y n.eff mayor que 100 para todas las estimaciones del modelo.

Todos los análisis fueron realizados de forma separada para hombres y mujeres, para el conjunto de la CAPV y para cada una de sus tres capitales. La comparación de la distribución de los valores de la RMEs entre los cuatro ámbitos geográficos se realizó mediante gráficos de cajas. Dado que en el caso de las capitales las defunciones esperadas Ei también fueron calculadas tomando como tasas de referencia las de la CAPV, la estimación del riesgo de una sección se puede descomponer como un producto del riesgo de la sección por pertenecer a la ciudad (este valor es común a todas las secciones que pertenecen a la misma ciudad) y el riesgo propio de la sección comparado con la ciudad a la que pertenece. Es este último el que se representa en los mapas de las capitales, de manera que las RME suavizadas ob- 
tenidas permiten valorar el riesgo de mortalidad de las secciones censales respecto al promedio general en dicha ciudad. La distribución geográfica de la RME suavizada derivada de los modelos BYM es representada mediante mapas categorizados en nueve cuantiles, siendo las áreas más oscuras las que corresponden a una RME más alta. Así mismo, el índice de privación es representado usando mapas categorizados en nueve cuantiles, en los que las áreas más claras son las más favorecidas, y las más oscuras las menos favorecidas. Todos los mapas fueron realizados mediante el paquete estadístico R.

\section{Resultados}

La tabla 1 describe la distribución del número de defunciones y de habitantes por secciones censales para la CAPV y las tres capitales. Durante el período 1996-2003 en todas las secciones se produjo alguna defunción en ambos sexos. En tres cuartas partes de las secciones se registraron al menos 27 defunciones en las mujeres y 34 en los hombres.

En 2001 las diferencias entre secciones censales en los indicadores socioeconómicos eran muy marcadas y de magnitud distinta según el ámbito geográfico (tabla 3). De las tres capitales, Bilbao mostró los valores más desfavorables y las mayores desigualdades sociales. De los cinco indicadores socioeconómicos, cabe destacar la variabilidad observada en la proporción de población joven con instrucción insuficiente, con coeficientes de variación ponderados por encima del $50 \%$ en todos los ámbitos geográficos; en el otro extremo, los valores del coeficiente de variación ponderada de la proporción de trabajadores eventuales estaban por debajo del 30\%.

La figura 1 muestra la distribución geográfica del índice de privación en las secciones censales de la CAPV y sus tres capitales. Las áreas menos favorecidas de la CAPV se concentraban principalmente en las regiones metropolitanas de Bilbao y Donostia-San Sebastián, y eran más frecuentes en Bizkaia y Gipuzkoa. Dentro de las capitales, el patrón socioeconómico era muy marcado. En Bilbao, las áreas más desfavorecidas se localizaban en el sur, y en los extremos este y oeste de la ciudad, mientras que las más favorecidas se situaban en el centro. En Donostia-San Sebastián, se daba un claro patrón esteoeste, de manera que las áreas menos favorecidas eran las de la mitad este de la ciudad. En Vitoria-Gasteiz, las secciones menos favorecidas se localizaban principalmente en el casco histórico y zonas aledañas del norte, así como en los extremos norte y sur de la ciudad. 
En ambos sexos la distribución de las RMEs de las secciones censales muestra una mayor mortalidad en Bilbao, seguida en orden de magnitud por Donostia-San Sebastián y Vitoria-Gasteiz (figura 2). Las diferencias entre las capitales fueron mayores en los hombres que en las mujeres, especialmente en el caso de Vitoria-Gasteiz respecto a las otras dos ciudades.

La figura 3 muestra que el riesgo de mortalidad en los hombres siguió un patrón geográfico similar al del índice de privación, tanto en el conjunto de la CAPV como en las tres capitales, de manera que las áreas con mayor privación tuvieron una mayor mortalidad. El patrón geográfico del riesgo de mortalidad en las mujeres mostró diferencias apreciables con el de los hombres (figura 4). Al igual que en los hombres, gran parte de las áreas con mayor privación tuvieron una mayor mortalidad. Sin embargo, en los tres ámbitos geográficos estudiados se observan áreas geográficas favorecidas con riesgos mayores de mortalidad en las mujeres. Así, es de notar la mayor mortalidad de las mujeres en áreas socioeconómicamente favorecidas al este de la ría del Nervión, o en el centro de las tres capitales.

El patrón de asociación entre la mortalidad y el índice de privación fue distinto según el sexo (tabla 3). En las mujeres, únicamente se observaron diferencias reseñables en los resultados relativos a la CAPV. Las mujeres residentes en las secciones correspondientes al quintil menos favorecido tuvieron una mortalidad un 7\% (Intervalo de credibilidad [I.C.] del 95\%: 3 $-12 \%$ ) mayor que las del quintil más favorecido. Además, si bien los intervalos de credibilidad incluyen el valor nulo (de 1) en todas las categorías, los resultados sugieren la existencia de una relación gradual entre el índice de privación y la mortalidad, con mayor mortalidad al aumentar el grado de privación. En los hombres, tanto en la CAPV como en las capitales se observaron diferencias mayores que en las mujeres. Así mismo, en la CAPV, en Bilbao y en Donostia-San Sebastián el RR de mortalidad seguía un claro gradiente socioeconómico, con mayor mortalidad al aumentar el grado de privación. En la CAPV, en los hombres la mortalidad en el quintil de secciones menos favorecidas fue un 23\% (I.C. del 95\%: 18-29) mayor que en las más favorecidas; los valores correspondientes a Bilbao y a San Sebastián fueron de un 34 (I.C. Del 95\%: 21-49) y de un 30\% (I.C. Del 95\%: 12-49). Por el contrario, en Vitoria-Gasteiz, si bien los intervalos de credibilidad incluyen el valor nulo, parece existir un exceso en la mortalidad en los dos últimos quintiles respecto al más favorecido. 
DisCUSIÓN

\section{Hallazgos principales}

Este estudio ha puesto en evidencia la existencia de desigualdades relevantes en la mortalidad total por áreas pequeñas en el País Vasco y en sus tres capitales, cuyas características difieren según el sexo y el ámbito geográfico. En ambos sexos, el riesgo de mortalidad y el índice de privación siguieron patrones geográficos similares, tanto en el conjunto de la CAPV como en sus tres capitales. En los hombres el riesgo de mortalidad mostraba una asociación positiva con el grado de privación socioeconómica y la magnitud de esa desigualdades era menor en Vitoria-Gasteiz que en los otros ámbitos geográficos. En las mujeres, por el contrario, unicamente se evidenciaron desigualdades socioeconómicas en la mortalidad en el conjunto de la CAPV.

\section{Patrón y magnitud de las desigualdades en la mortalidad en áreas pequeñas}

El patrón de asociación positiva entre privación socioeconómica del lugar de residencia y mortalidad aparece de forma consistente en los diversos lugares en que se ha estudiado (Carstairs y Morris, 1991; Chen et al., 2006; Diez-Roux et al., 2007). Así se ha evidenciado en los estudios relativos a España o sus regiones, ya utilizaran el municipio o agregados de municipios (Benach et al., 1999, Benach et al., 2002), o la sección censal (Esnaola et al., 2006) como unidad de análisis. Así mismo, los estudios de áreas urbanas han mostrado ese mismo patrón, bien utilizando los barrios o las zonas básicas de salud como unidades de análisis (Borrell y Arias, 1993; Pasarín et al., 1999; Ruiz-Ramos et al., 2004), o cuando se han basado en datos más desagregados por secciones censales (Ocaña et al., 2009; Domínguez-Berjón et al., 2005; Cano-Serral et al., 2009).

Los resultados de este estudio también concuerdan con la experiencia previa en lo relativo a la magnitud de las desigualdades socioeconómicas en la mortalidad, y a las diferencias en las desigualdades entre hombres y mujeres (Ocaña et al., 2009; Cano-Serral et al., 2009). Estas diferencias entre sexos podrían explicarse por las distintas exposiciones en el ámbito laboral, así como por las diferencias en las conductas relacionadas con la salud. Además, se ha argumentado que los indicadores socioeconómicos utilizados podrían no ser adecuados para valorar la privación socioeconómica en las mujeres (Borrell et al., 2004). 


\section{Fortalezas y limitaciones}

Entre las fortalezas del estudio se incluye el uso de áreas pequeñas (sección censal) y de métodos estadísticos basados en la suavización de las tasas de mortalidad. Las secciones censales son áreas pequeñas, relativamente homogéneas respecto a sus características socioeconómicas, de las que se dispone de información censal fácilmente accesible. Los estudios basados en áreas pequeñas pueden detectar patrones geográficos y socioeconómicos en la mortalidad no identificables en estudios basados en áreas de mayor tamaño (Waller y Gotway, 2004). Los métodos estadísticos de epidemiología espacial utilizados, además de considerar la estructura espacial de los datos, evitan que el patrón geográfico esté artificialmente dominado por regiones poco pobladas donde la estimación de los riesgos es generalmente más extrema y menos fiable (Elliot y Wartenberg, 2004). Así mismo, se trata del primer estudio en España de estas características que incluye una comunidad autónoma y sus capitales.

Una de las limitaciones del estudio viene impuesta por las características temporales de los datos. Los indicadores socioeconómicos de 2001 pudieran no reflejar de forma adecuada las características socioeconómicas de las áreas para todo el período de estudio. De manera similar, la población residente en las secciones censales podría haber cambiado a lo largo de los años. Además, cobran especial importancia los sesgos inducidos por los cambios de población asociados a la migración no registrada (Ocaña-Riola, 2009). Se ha estimado que el Censo de 2001 podría subestimar la población inmigrante hasta en un 16\% (Devolder et al., 2006). En el País Vasco, la magnitud de las migraciones no era relevante durante el período de estudio (Aierdi, 2006), por lo que el efecto potencial de la migración no registrada sería pequeño. Otra limitación tiene que ver con el uso de datos de mortalidad de varios años agregados en el tiempo y analizados mediante modelos que no consideran la dependencia temporal de los datos, lo que podría inducir a sesgos en las estimaciones del riesgo de mortalidad en estudios de áreas pequeñas (Ocaña-Riola, 2007).

\section{Interpretación de los resultados e implicaciones prácticas}

La asociación ecológica entre la privación socioeconómica del área de residencia y la mortalidad probablemente refleja una combinación del efecto individual (composicional) ligado a la posición socioeconómica y del efecto de factores contextuales relacionados con el medioambiente social y físico del área (Diez-Roux et al., 2007). La extensión del análisis presentado a las princi- 
pales causas de mortalidad sería un elemento de gran ayuda para sugerir los factores implicados en la génesis de esas desigualdades.

La magnitud de las desigualdades socioeconómicas mostradas en este estudio ilustra la relevancia de las desigualdades sociales en salud en nuestro contexto. Razones de justicia y de equidad han llevado a considerar el fenómeno de las desigualdades sociales en salud como una prioridad de las políticas públicas (CSDH, 2008). Algunos países de nuestro entorno ya han desarrollado políticas específicas para reducir las desigualdades en salud (Swedish National Institute of Public Health, 2003; Norwegian Ministry of Health and Care Services, 2006; Ministry of Social Affairs and Health, 2008). Así mismo, el Plan de Salud del País Vasco 2002-2010 incluyo entre sus prioridades la reducción de las desigualdades sociales en salud (Departamento de Sanidad, 2002). La monitorización de las desigualdades sociales en salud es un elemento clave para iniciar y desarrollar esas políticas. En este sentido, los estudios como el mostrado, basados en el uso de información socioeconómica del área de residencia, permiten valorar la magnitud de las desigualdades sociales en salud e identificar las áreas geográficas con mayores necesidades de intervención.

Recibido: 29/06/2009

Aceptado: 30/09/2009

\section{BiBLIOGRAFÍA}

Aierdi, X.: "Población extranjera". Eustat. Informe socioeconómico de la C.A de Euskadi 2006. Eustat-Instituto Vasco de Estadística. Vitoria-Gasteiz, 2006.

Barceló, M. A., Saez, M., Cano-Serral. G., Martínez-Beneito. M. A., Martínez, J. M., Borrell, C. et al.: "Métodos de suavización de indicadores de mortalidad: aplicación al análisis de las desigualdades en la mortalidad en ciudades de España (Proyecto MEDEA)". Gac Sanit 2008; 22(6):596-608.

Benach, J., Yasui, Y.: "Geographical patterns of excess mortality in Spain explained by two indices of deprivation". J Epidemiol Community Health 1999; 53(7):423-431.

Benach, J., Martínez, J. M., Borrell, C., Pasarín, M. I., Yasui, Y.: "Desigualtats geogràfiques en àrees petites". En Borrell, C., Benach, J. (eds.). Les desigualtats en la salut a Catalunya. Editorial Mediterrània, S.L., Barcelona, 2002.

Benach, J., Yasui, Y., Borrell, C., Pasarin, M. I., Martinez, J. M., Daponte, A.: "The public health burden of material deprivation: excess mortality in leading causes of death in Spain". Prev Med 2003; 36(3):300-308.

Besag, J., York, J., Mollie, A.: "Bayesian image restoration, with two applications in spatial statistics". Annals of the Institute of Statistical Mathematics. 1991; 43:1-59. 
Best, N. G., Richardson, S., Thomson, A.: "A comparison of Bayesian spatial models for disease mapping". Stat Methods Med Res 2005; 14:35-59

Borrell, C., Arias, A.: "Desigualtats de mortalitat en els barris de Barcelona 1983-89". Gac Sanit 1993; 38:205-0.

Borrell, C., Rohlfs, I., Artazcoz, L., Muntaner, C.: "Desigualdades en salud según la clase social en las mujeres. ¿Cómo influye el tipo de medida de la clase social?". Gac Sanit 2004; 18 Supl 2: 75-82.

Brooks, S. P., Gelman, A.: "Alternatives methods for monitoring convergence of iterative simulations". Journal of Computational and Graphical Statistics 1998; $7: 434-455$

Cano-Serral, G., Azlor, E., Rodríguez-Sanz, M., Pasarín, M. I., Martínez, J. M., Puigpinós, R., et al.: "Socioeconomic inequalities in mortality in Barcelona: A study based on census tracts (MEDEA Project)". Health and Place 2009; 15:186-192.

Carstairs, V., Morris, R.: Deprivation and health in Scotland. Aberdeen University Press, Aberdeen, 1991.

Chen, J. T., Rehkopf, D.H., Waterman, P. D., Subramanian, S. V., Coull, B. A., Cohen, B., Ostrem, M., Krieger, N.: "Mapping and measuring social disparities in premature mortality: the impact of census tract poverty within and across Boston neighborhoods, 1999-2001". J Urban Health. 2006 Nov; 83(6):1063-84.

Clayton, D., Kaldor, J.: "Empirical Bayes estimates for age-standardized relative risks". Biometrics 1987; 43:671-681.

CSDH: Closing the gap in a generation: health equity through action on the social determinants of health. Final Report of the Commission on Social Determinants of Health. World Health Organization, Geneva, 2008.

Departamento de Sanidad: Política de salud para Euskadi. Plan de Salud 2002-2010. Servicio Central de Publicaciones del Gobierno Vasco. Vitoria-Gasteiz, 2002.

Devolder, D., Gil, F., Forte, P.: "Estimación del grado de error en el registro de la población extranjera en España: un enfoque comparativo". PAPERS DE DEMOGRAFIA 2006; 309: 1-16.

Diez-Roux, A. V.: "Investigating neighborhood and area effects on health". Am J Public Health 2001;91:1783-9.

Diez-Roux, A. V., Franklin, T. G., Alazraqui, M., Spinelli, H.: "Intraurban variations in adult mortality in a large latin american city". J Urban Health 2007; 84: 319-333.

Diez-Roux, A. V.: "Neighborhoods and health: where are we and were do we go from here?". Rev Epidemiol Santé Publique 2007; 55(1):13-21.

Domínguez-Berjón, M. F., Borrell, C., López, R., Pastor, V.: "Mortality and socioeconomic deprivation in census tracts of an urban setting in southern Europe". J Urban Health 2005; 82:225-36.

Domínguez-Berjón, M. F., Borrell, C., Cano-Serral, G., Esnaola, S., Nolasco, A., Pasarín, M. I., Ramis, R., Saurina, C., Escolar-Pujolar, A.: "Construcción de un índice de privación basado en datos censales in grandes ciudades de España (Proyecto MEDEA)". Gac Sanit. 2008; 22:179-87. 
Elliot, P., Wartenberg, D.: "Spatial Epidemiology: Current Approaches and Future Challenges". Environmental Health Perspectives 2004; 112(9):998-1006.

English, D.: "Geographical epidemiology and ecological studies". En Elliott, P., Cuzick, J., English, D., Stern, R. (eds.). Geographical and environmental epidemiology. Methods for small-area studies.Oxford University Press, New York, 1992.

Esnaola, S., Aldasoro, E., Ruiz, R., et al.: "Desigualdades socioeconómicas en la mortalidad en la Comunidad Autónoma del País Vasco". Gac Sanit 2006; 20:16-24.

Gelman, A.: "Prior distributions for variance parameters in hierarchical models". Bayesian Analysis 2006; 3:515-533

Kawachi, I., Berkman, L. F.: "Introduction". En Kawachi, I., Berkman, L. F. (eds.). Neighborhoods and health. Oxford University Press, New York, 2003.

Krieger, N.: "A century of census tracts: health and the body politic (1906-2006)" J Urban Health 2006; 83(3):355-61.

Lunn, D. J., Thomas, A., Best, N., Spiegelhalter, D.: "WinBUGS - a Bayesian modelling framework: concepts, structure, and extensibility". Statistics and Computing 2000; 10:325-337.

Martínez Beneito, M. A., López Quilez, A., Amador Iscla, A., Melchor Alós, I., Botella Rocamora, P., Abellán Andrés, C. et al.: Atlas de Mortalidad de la Comunitat Valenciana 1991-2000. Conselleria de Sanitat, Generalitat Valenciana, Valencia, 2005.

Macintyre, S., Elleway, A.: "Ecological approaches: rediscovering the role of the physical and social environment". En: Berkman, L., Kawachi, I. (eds.). Social epidemiology. Oxford: Oxford University Press, 2000.

Ministry of Social Affairs and Health: National Action Plan to Reduce Health Inequalities 2008-2011. Helsinki: Publications of the Ministry of Social Affairs and Health; 2008

Nolasco, A., Melchor, I., Moncho, J., García, C., Verdú, J., Caballero, P. et al.: "Vigilancia de la mortalidad en ciudades: resultados en Valencia y Alicante". Gac Sanit 2004;18:7-15.

Norwegian Ministry of Health and Care Services. National strategy to reduce social inequalities in health. Report No. 20 (2006-2007) to the Storting. Norwegian Ministry of Health and Care Services; 2006.

Ocaña-Riola, R.: "The misuse of count data aggregated over time for disease mapping". Statist. Med. 2007; 26:4489-4504.

Ocaña-Riola, R., Saurina, C., Fernández-Ajuria, A., Lertxundi, A., Sánchez-Cantalejo, C., Saez, M., et al.: "Area deprivation and mortality in the provincial capital cities of Andalusia and Catalonia (Spain)". J Epidemiol Comm Health 2008; 62:147-152.

Ocaña-Riola, R., Fernández-Ajuria, A., Mayoral-Cortés, J. M., Toro-Cárdenas, S., Sánchez-Cantalejo, C.: "Uncontrolled migrations as a cause of inequality in health and mortality in small-area studies". Epidemiology 2009; 20:411-18.

Pasarín, I., Borrell, C., Plasència A.: " ¿Dos patrones de desigualdades sociales en mortalidad en Barcelona?”. Gac Sanit 1999; 13:431-440. 
Phillimore, P., Beattie, A., Townsend, P.: "Widening inequality of health in northern England, 1981-91". BMJ 1994; 308:1125-8.

$R$ Development Core Team. R: A language and environment for statistical computing. $\mathrm{R}$ Foundation for Statistical Computing, Vienna, Austria, 2007, ISBN 3-900051-07-0, http://www.R-project.org.

Ruiz-Ramos, M., Sánchez, J., Garrucho, G., Viciana, F.: "Desigualdades en mortalidad en la ciudad de Sevilla". Gac Sanit 2004; 18:16-23.

Susser, M., Susser, E.: "Choosing a future for epidemiology: II. From black box to Chinese boxes and eco-epidemiology". Am J Public Health 1996; 86:674-7.

Swedish National Institute of Public Health: Sweden's new public health policy. National public health objectives for Sweden. Swedish National Institute of Public Health; 2003.

Wallace, R., Wallace, D.: "Socioeconomic determinants of health: community marginalisation and the difusión of disease and disorder in the United States". BMJ 1997; 314:1341-5.

Waller, L., Gotway, C.: Applied spatial statistics for public health data. Wiley; Hoboken, 2004.

\section{RESUMEN}

Se describen las desigualdades socioeconómicas en la mortalidad por secciones censales en el País Vasco y en sus tres capitales durante el período 1996-2003. Se realizó un estudio ecológico transversal de áreas pequeñas. Mediante el análisis de componentes principales se calculó un índice de privación socioeconómica. El riesgo de mortalidad de cada sección censal, y la relación entre la mortalidad y la privación socioeconómica se estudiaron mediante modelos Bayesianos jerárquicos. Los patrones geográficos del riesgo de mortalidad y del índice de privación fueron similares, con mayor mortalidad en las áreas de mayor privación. En el conjunto del País Vasco la magnitud de la asociación entre la privación y la mortalidad fue mayor en los hombres que en las mujeres. En las capitales esa asociación sólo se evidenció para los hombres. Estos resultados son útiles para orientar las políticas de salud e identificar las áreas geográficas que precisan una mayor atención.

Palabras ClaVe: factores socioeconómicos; desigualdades en salud; privación; mortalidad; análisis de áreas pequeñas.

\section{ABSTRACT}

Census track level socioeconomic inequalities in mortality are described in the Basque Country and its three main cities in the 1996-2003 period. An ecological crosssectional study of small areas was performed. Using principal component analysis a socioeconomic deprivation index was calculated. The mortality risk for each census track and the relation between mortality and socioeconomic deprivation were studied 
by means of hierarchical Bayesian models. Geographical patterns of the mortality risk and the deprivation index were similar, with higher mortality in more deprived areas. In the Basque Country as a whole the magnitude of the association between mortality and deprivation was higher in men than in women. In the cities, the association was only apparent for men. These results are useful to guide health policies and identify geographical areas with greater needs.

KEY WORDS: socioeconomic factors; health inequalities; deprivation; mortality; smallarea analysis.

\section{RÉSUMÉ}

On décrit les inégalités socio-économiques dans la mortalité par sections de recensement au Pays Basque et ses trois capitales pendant la période 1996-2003. Une étude écologique transversale d'aires petites a été accomplie. Au moyen de l'analyse de composantes principales, on a calculé un index de privation socio-économique. Le risque de mortalité de chaque section, et le rapport entre la mortalité et la privation socioéconomique ont été étudiés utilisant des modèles Bayesians hiérarchiques. Les patrons géographiques du risque de mortalité et de l'index de privation étaient semblables, étant la mortalité plus grande dans les aires avec une plus grande privation. Dans l'ensemble du Pays Basque la grandeur de l'association entre la privation et la mortalité était plus grande chez les hommes que chez les femmes. Dans les capitales l'association était evidente seulement chez les hommes. Ces résultats sont utiles pour orienter les politiques de santé et pour identifier les aires géographiques qui ont besoin d'une plus grande attention.

MoTS CLÉs: facteurs socio-économiques; inégalités en santé; privation; mortalité; analyse d'aires petites. 
TABLA 1

DISTRIBUCIÓN DEL NÚMERO DE DEFUNCIONES (HABITANTES) POR SECCIONES CENSALES

\begin{tabular}{|c|c|c|c|c|c|c|c|}
\hline $\begin{array}{l}\text { SEXO } \\
\text { Ambito geográfico }\end{array}$ & Minímo & $\mathrm{P} 25^{\mathrm{a}}$ & $\mathrm{P} 50$ & Media & P75 & Máximo & Total \\
\hline \multicolumn{8}{|l|}{ Mujeres } \\
\hline $\mathrm{CAPV}^{\mathrm{b}}$ & $1(242)$ & $27(482)$ & $36(618)$ & $41(647)$ & $51(788)$ & $243(1.628)$ & $68.246(1.064 .704)$ \\
\hline Bilbao & $11(278)$ & $32(476)$ & $42(627)$ & $47(640)$ & $57(781)$ & $205(1.202)$ & 13.602 (184.310) \\
\hline Donostia-San Sebastián & $9(334)$ & $31(544)$ & $48(648)$ & $51(676)$ & $65(799)$ & $243(1.215)$ & $(94.577)$ \\
\hline Vitoria-Gasteiz & $4(321)$ & $20(491)$ & $30(635)$ & $35(657)$ & $45(791)$ & $226(1.606)$ & $5.935 \quad(110.424)$ \\
\hline \multicolumn{8}{|l|}{ Hombres } \\
\hline CAPV & $4(236)$ & $34(458)$ & $45(588)$ & $47(619)$ & $58(754)$ & $169(1.734)$ & 77.949 (1.017.883) \\
\hline Bilbao & $15(243)$ & $39(416)$ & $48(554)$ & $51(575)$ & $61(713)$ & $158(1.107)$ & $14.668 \quad(165.662)$ \\
\hline Donostia-San Sebastián & $14(276)$ & $35(440)$ & $45(578)$ & $48(599)$ & $59(739)$ & $135(1.144)$ & $(83.800)$ \\
\hline Vitoria-Gasteiz & $4(263)$ & $26(466)$ & $38(608)$ & $40(634)$ & $53(767)$ & $100(1.734)$ & $(106.428)$ \\
\hline
\end{tabular}

a Percentil 25

b Comunidad Autónoma del País Vasco

TABLA 2

DESIGUALDADES EN LA MORTALIDAD TOTAL SEGÚN PRIVACIÓN SOCIOECONÓMICA EN LA CAPVª Y SUS CAPITALES, 1996-2003

\begin{tabular}{|c|c|c|c|c|c|c|c|c|}
\hline \multirow{2}{*}{$\begin{array}{l}\text { SEXO } \\
\text { Indice de } \\
\text { privación }\end{array}$} & \multicolumn{2}{|r|}{ CAPV } & \multicolumn{2}{|r|}{ Bilbao } & \multicolumn{2}{|c|}{$\begin{array}{c}\text { Donostia- } \\
\text { San Sebastián }\end{array}$} & \multicolumn{2}{|c|}{ Vitoria-Gasteiz } \\
\hline & $\mathrm{RR}$ & (I.C. del 95\%) & $\mathrm{RR}$ & (I.C. del 95\%) & $\mathrm{RR}$ & (I.C. del 95\%) & $\mathrm{RR}$ & (I.C. del 95\%) \\
\hline \multicolumn{9}{|l|}{ Mujeres } \\
\hline $\mathrm{Q} 1$ & 1 & & 1 & & 1 & & 1 & \\
\hline Q2 & 0,99 & $(0,95-1,03)$ & 0,96 & $(0,88-1,06)$ & 1,08 & $(0,93-1,24)$ & 0,92 & $(0,77-1,09)$ \\
\hline Q3 & 1,01 & $(0,97-1,05)$ & 0,99 & $(0,89-1,10)$ & 1,07 & $(0,92-1,24)$ & 0,96 & $(0,81-1,13)$ \\
\hline Q4 & 1,03 & $(0,99-1,08)$ & 0,97 & $(0,87-1,08)$ & 1,01 & $(0,85-1,20)$ & 1,02 & $(0,87-1,20)$ \\
\hline Q5 & 1,07 & $(1,03-1,12)$ & 1,00 & $(0,89-1,13)$ & 1,08 & $(0,88-1,29)$ & 0,99 & $(0,83-1,17)$ \\
\hline \multicolumn{9}{|l|}{ Hombres } \\
\hline Q1 & 1 & & 1 & 1 & & & 1 & \\
\hline Q2 & 1,07 & $(1,03-1,11)$ & 1,03 & $(0,94-1,13)$ & 1,08 & $(0,96-1,21)$ & 0,99 & $(0,86-1,16)$ \\
\hline Q3 & 1,12 & $(1,07-1,16)$ & 1,12 & $(1,02-1,24)$ & 1,20 & $(1,06-1,34)$ & 1,01 & $(0,86-1,16)$ \\
\hline Q4 & 1,16 & $(1,11-1,2)$ & 1,16 & $(1,05-1,29)$ & 1,16 & $(1,02-1,31)$ & 1,14 & $(0,98-1,33)$ \\
\hline Q5 & 1,23 & $(1,18-1,29)$ & 1,34 & $(1,21-1,49)$ & 1,30 & $(1,12-1,49)$ & 1,14 & $(0,97-1,33)$ \\
\hline
\end{tabular}

a Comunidad Autónoma del País Vasco.

b Quintiles del índice de privación.

RR: Riesgo relativo. I.C.: Intervalo de credibilidad 
TABLA 3

VALORESa PROMEDIO DE LOS INDICADORES SOCIOECONÓMICOS SEGÚN LOS QUINTILES DEL ÍNDICE DE PRIVACIÓN

\begin{tabular}{|c|c|c|c|c|c|}
\hline AMBITO GEOGRÁFICO & En desempleo & $\begin{array}{l}\text { Instrucción } \\
\text { insuficiente }\end{array}$ & $\begin{array}{l}\text { Instrucción } \\
\text { insuficiente } \\
\text { población } \\
\text { joven }\end{array}$ & $\begin{array}{c}\text { Trabajadores } \\
\text { trabajadoras } \\
\text { manuales }\end{array}$ & $\begin{array}{c}\text { Trabajadores } \\
\text { trabajadoras } \\
\text { eventuales }\end{array}$ \\
\hline \multicolumn{6}{|l|}{$\mathrm{CAPV}^{\mathrm{c}}$} \\
\hline Q1 & 9,6 & 15,7 & 4,8 & 32,4 & 15,8 \\
\hline Q2 & 10,2 & 24,3 & 6,1 & 52,3 & 20,2 \\
\hline Q3 & 11,0 & 29,6 & 7,4 & 60,9 & 23,1 \\
\hline Q4 & 12,3 & 35,4 & 9,1 & 66,4 & 25,3 \\
\hline Q5 & 16,3 & 40,7 & 15,7 & 71,9 & 30,0 \\
\hline Total & 11,8 & 28,7 & 8,4 & 56,1 & 22,6 \\
\hline $\mathrm{CV}^{\mathrm{d}}(\%)$ & 37,2 & 37,8 & 66,1 & 30,3 & 26,8 \\
\hline \multicolumn{6}{|l|}{ Bilbao } \\
\hline Q1 & 10,7 & 13,6 & 4,3 & 20,2 & 13,8 \\
\hline Q2 & 12,4 & 22,2 & 6,1 & 34,4 & 17,6 \\
\hline Q3 & 14,6 & 28,8 & 8,2 & 47,2 & 21,4 \\
\hline Q4 & 16,0 & 35,9 & 11,2 & 60,2 & 24,2 \\
\hline Q5 & 21,6 & 45,4 & 22,8 & 71,2 & 28,6 \\
\hline Total & 15,0 & 28,9 & 10,3 & 46,3 & 21,0 \\
\hline CV (\%) & 33,3 & 41,5 & 79,8 & 41,9 & 27,8 \\
\hline \multicolumn{6}{|l|}{ Donostia-San Sebastián } \\
\hline Q1 & 8,5 & 13,7 & 4,7 & 25,5 & 14,2 \\
\hline Q2 & 11,3 & 19,0 & 6,7 & 30,4 & 16,4 \\
\hline Q3 & 11,9 & 24,8 & 7,6 & 40,8 & 19,5 \\
\hline Q4 & 12,9 & 31,9 & 8,7 & 49,5 & 21,9 \\
\hline Q5 & 13,6 & 38,6 & 13,1 & 69,0 & 27,1 \\
\hline Total & 11,5 & 25,1 & 8,0 & 42,4 & 19,6 \\
\hline CV (\%) & 23,0 & 42,2 & 53,5 & 39,5 & 27,6 \\
\hline \multicolumn{6}{|l|}{ Vitoria-Gasteiz } \\
\hline Q1 & 7,3 & 15,1 & 5,4 & 32,7 & 15,1 \\
\hline Q2 & 9,1 & 20,7 & 8,0 & 53,4 & 20,7 \\
\hline Q3 & 9,7 & 29,0 & 9,9 & 61,2 & 22,7 \\
\hline Q4 & 10,8 & 36,2 & 11,9 & 65,4 & 24,6 \\
\hline Q5 & 13,3 & 41,5 & 17,7 & 70,2 & 28,5 \\
\hline Total & 9,9 & 27,5 & 10,2 & 55,6 & 21,9 \\
\hline $\mathrm{CV}(\%)$ & 26,6 & 40,0 & 59,3 & 27,9 & 23,5 \\
\hline
\end{tabular}

a Promedio ponderado de los valores porcentuales correspondientes a las secciones censales incluidas en ese quintil

${ }^{b}$ Quintiles del índice de privación.

${ }^{\mathrm{c}}$ Comunidad Autónoma del País Vasco

d Coeficiente de variación ponderado por el tamaño poblacional 


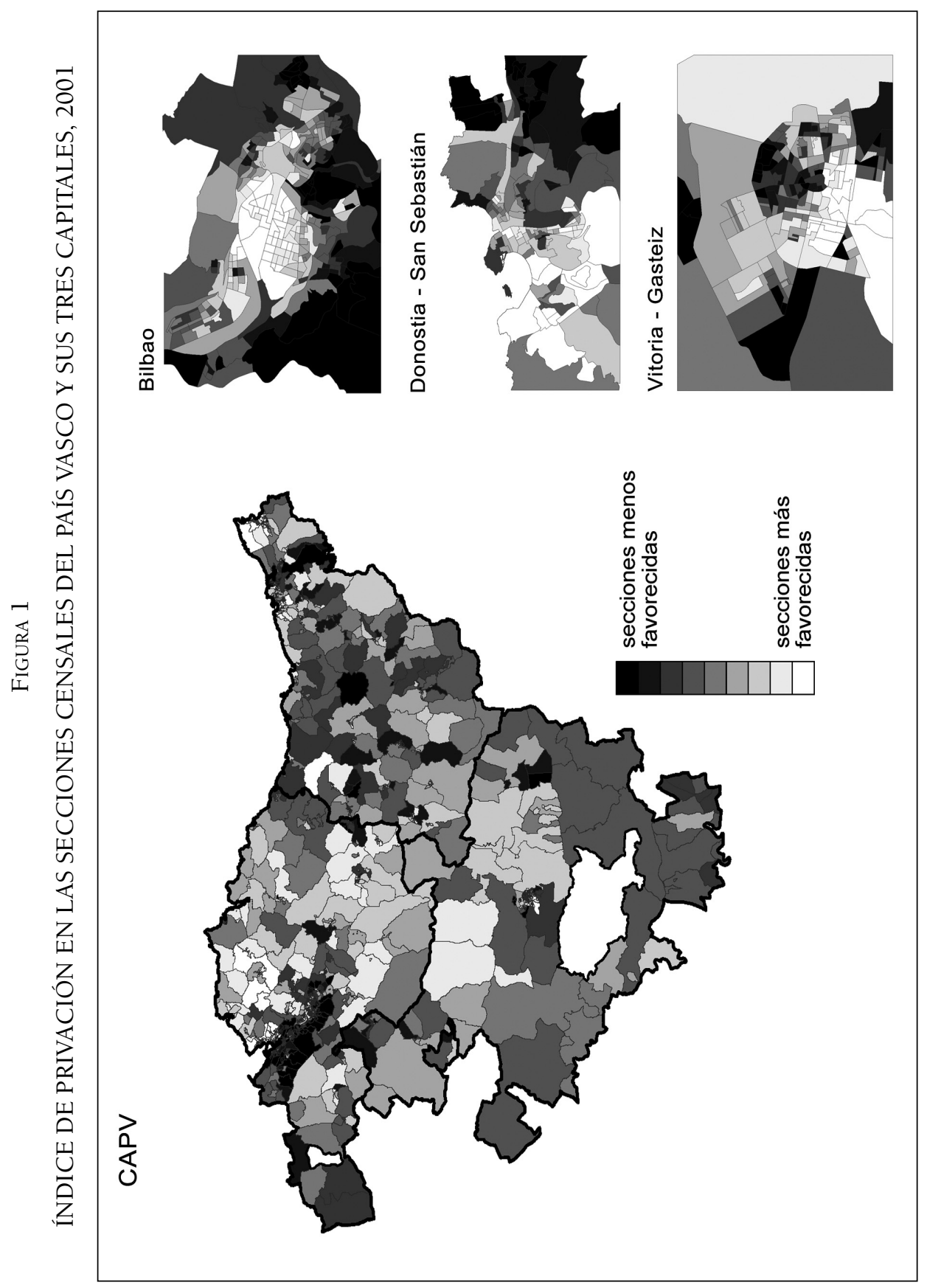

Estudios Geográficos, Vol. LXX, 267, pp. 443-462, julio-diciembre 2009 ISSN: 0014-1496, eISSN: 1988-8546, doi: 10.3989/estgeogr.0468 


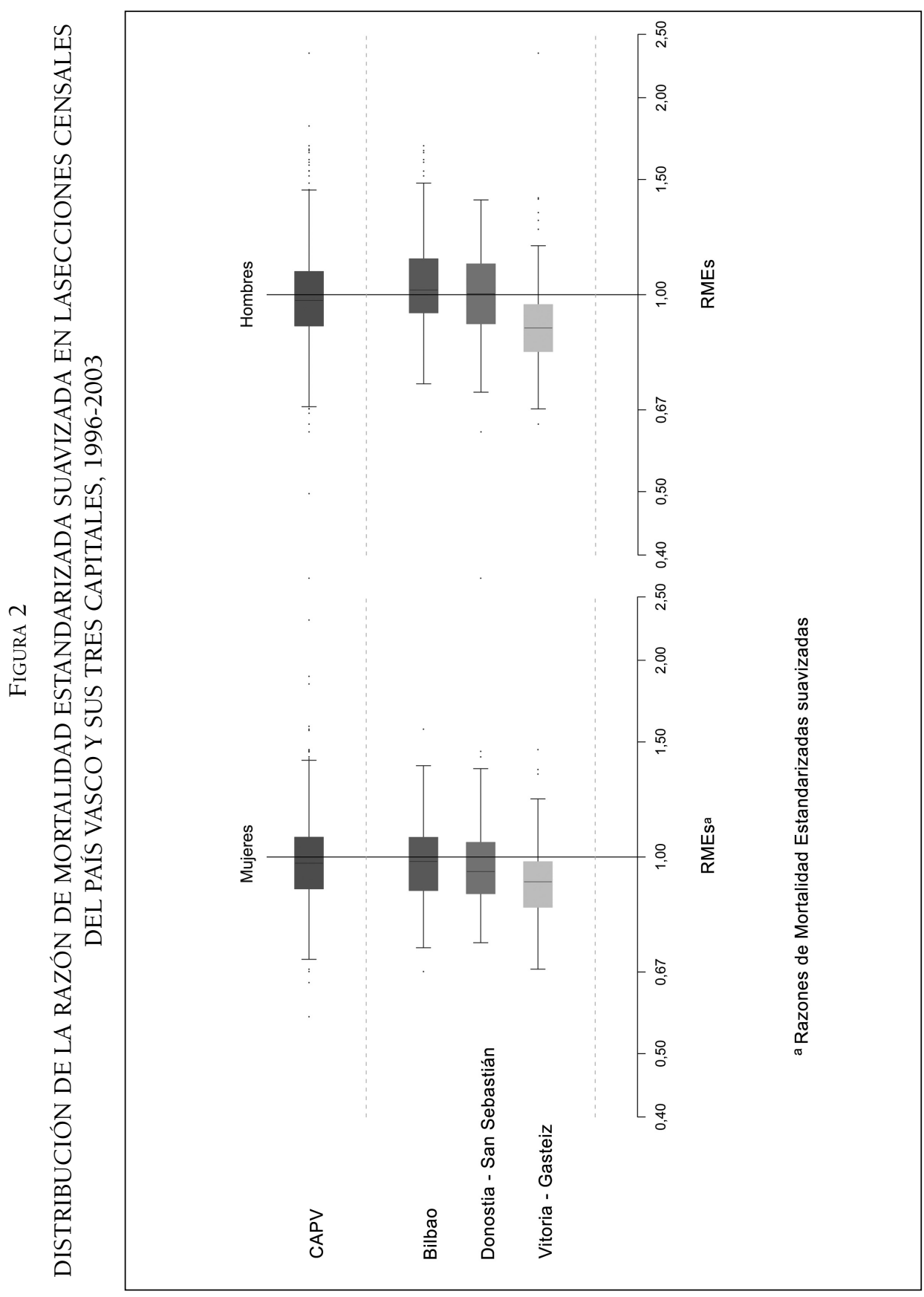

Estudios Geográficos, Vol. LXX, 267, pp. 443-462, julio-diciembre 2009 ISSN: 0014-1496, eISSN: 1988-8546, doi: 10.3989/estgeogr.0468 


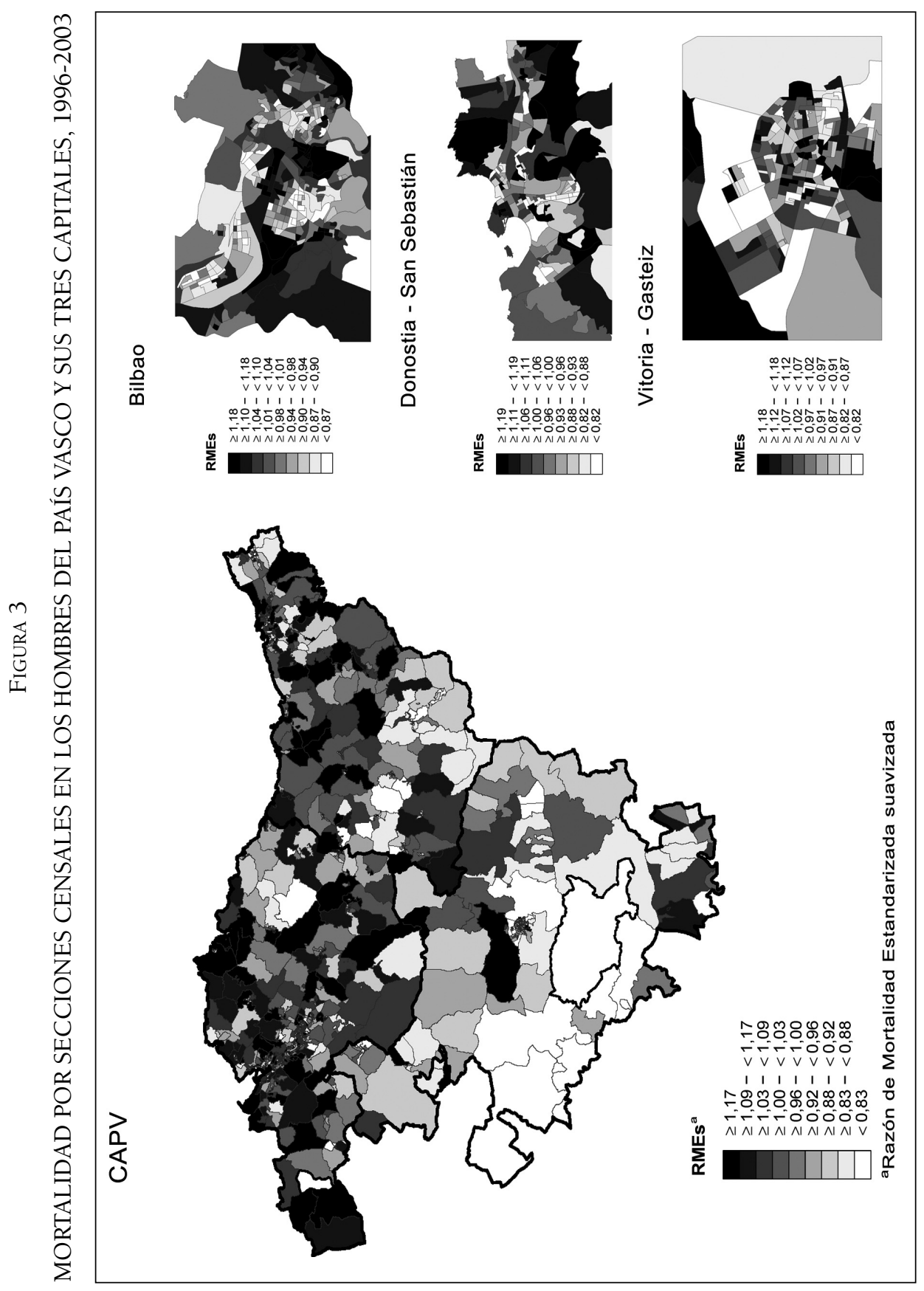

Estudios Geográficos, Vol. LXX, 267, pp. 443-462, julio-diciembre 2009 ISSN: 0014-1496, eISSN: 1988-8546, doi: 10.3989/estgeogr.0468 


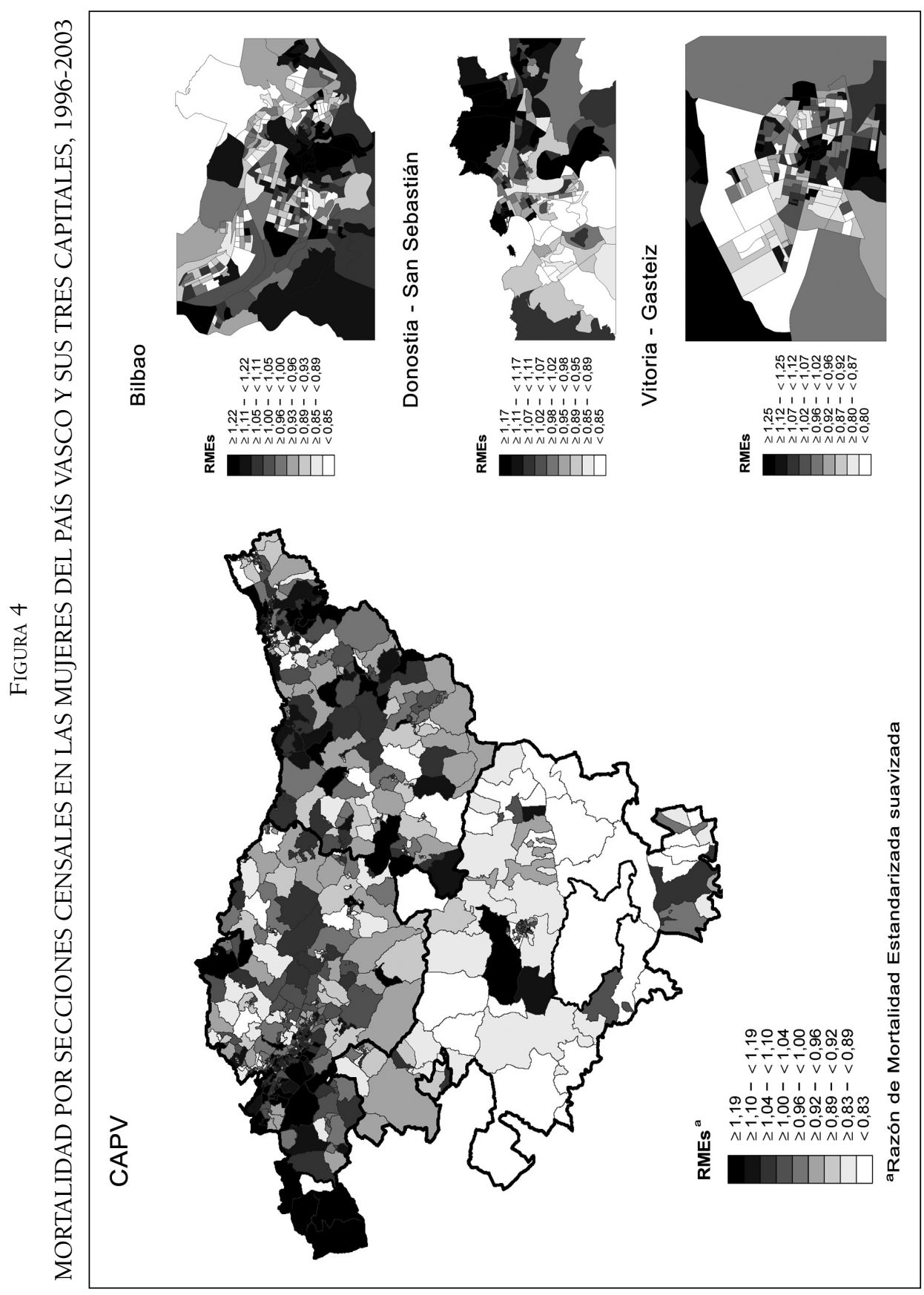

Estudios Geográficos, Vol. LXX, 267, pp. 443-462, julio-diciembre 2009 ISSN: 0014-1496, eISSN: 1988-8546, doi: 10.3989/estgeogr.0468 